\title{
MULTICUBE: Multi-Objective Design Space Exploration of Multi-Core Architectures
}

\author{
Cristina Silvano*, William Fornaciari*, Gianluca Palermo*, Vittorio Zaccaria*, Fabrizio Castro*, \\ Marcos Martinez $^{\dagger}$, Sara Bocchio ${ }^{\ddagger}$, Roberto Zafalon ${ }^{\ddagger}$, Prabhat Avasare ${ }^{\S}$, Geert Vanmeerbeeck ${ }^{\S}$,

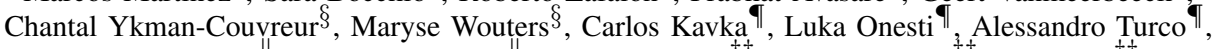 \\ Umberto Bondi ${ }^{l}$, Giovanni Mariani ${ }_{x}$, Hector Posadas ${ }^{\ddagger \ddagger}$, Eugenio Villar ${ }_{x}^{\ddagger \ddagger}$, Chris $\mathrm{Wu}^{\dagger \dagger}$, \\ Fan Dongrui , Zhang Hao ${ }^{\mathrm{x}}$, Tang Shibin \\ * Dipartimento di Elettronica e Informazione - Politecnico di Milano - Milano - Italy \\ ${ }^{\dagger}$ Design of Systems on Silicon (DS2) - Valencia - Spain, ${ }^{\ddagger}$ STMicroelectronics - Italy, ${ }^{\S}$ IMEC vZw - Belgium,

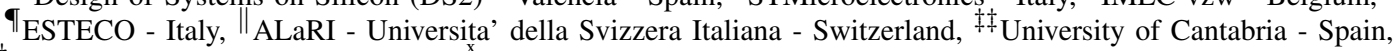 \\ ${ }^{\dagger}$ STMicroelectronics Beijing - China, ${ }^{\mathrm{x}}$ Institute of Computing Technology - Chinese Academy of Sciences - China
}

\begin{abstract}
Technology trends enable the integration of many processor cores in a System-on-Chip (SoC). In these complex architectures, several architectural parameters can be tuned to find the best trade-off in terms of multiple metrics such as energy and delay. The main goal of the MULTICUBE project consists of the definition of an automatic Design Space Exploration framework to support the design of next generation many-core architectures $^{1}$.
\end{abstract}

\section{INTRODUCTION}

The main goal of MULTICUBE project is the definition of an automatic framework to support multi-objective Design Space Exploration (DSE) of multi and many core SoC architectures. The framework enables the tuning of several architectural parameters to minimize multiple metrics (such as energy and latency) while meeting system-level constraints (such as throughput, bandwidth and QoS). The project focuses on the definition of an automatic modeling and optimization methodologies for improving the conventional SoC design flow. In such a design flow, the optimization process is still done manually, based on the past experience of the designer to leverage existing simulation models to explore the design space and find out power-performance trade-offs (Pareto analysis).

The project targets an efficient and automatic exploration of parallel embedded architectures in terms of several design parameters such as available parallelism (e.g., number of cores, processor issue width), cache-subsystem (e.g. cache size and associativity) and NoC-related parameters (e.g., channel buffer size). When dealing with complex many-core architectures, the design space exploration can easily become very huge, making a full-search simulation-based exploration unfeasible. Automatic exploration techniques based on optimisation heuristics should be used to figure out an approximate Pareto set in a reasonable time. The exploration time can even be reduced by decreasing the number of design points to be simulated. This can be done by using analytical models to predict the system behavior corresponding to design points not yet simulated.

\footnotetext{
${ }^{1}$ This project is supported by the EC under grant MULTICUBE FP7-216693
}

These analytical models are defined from a training set of simulations.

\section{THE MULTICUBE DESIGN METHODOLOGY}

The MULTICUBE design flow (shown in Figure 1) consists of two frameworks:

Power/Performance Estimation Framework includes the simulation tools for power and performance estimation of the target architectures to be explored. Each configurable simulation model accepts an architectural configuration as input and generates, at the end of simulation, the corresponding system-level metrics running the reference application. The combination of architecture and reference application is defined, from here on, as use case. The simulators used in the context of the project are either open source (MULTICUBESCOPE) or proprietary (such as the IMEC TLM platform, the STMicroelectronics SP2 simulator or the Institute of Computing Technology's (ICT) many core simulator). The tool interface has been standardized with the definition of the MULTICUBE XML interface so as other simulators can be plugged in with minimal effort. Besides event-based simulation, a set of Response Surface Models has been developed to further speedup the evaluation of the system-level metrics through analytical expressions derived from a training set of simulations.

Design Space Exploration Framework contains the tools for automatic DSE and interacts with the power/performance estimation framework by iteratively generating different instances of the design space and reading back power and performance evaluation metrics (system metrics). This framework includes several optimization heuristics for identifying power/performance trade-offs and generating the approximated Pareto frontier which may be more or less close to the actual Pareto front. The DSE tools developed in the project are: the open source (MULTICUBE Explorer tool) and the proprietary (ESTECO's modeFRONTIER) optimization tool (widely used tool in multi-disciplinary optimization).

The Use Case and Simulator Provider. (S)he is the system architect providing the simulator with a set of configurable 


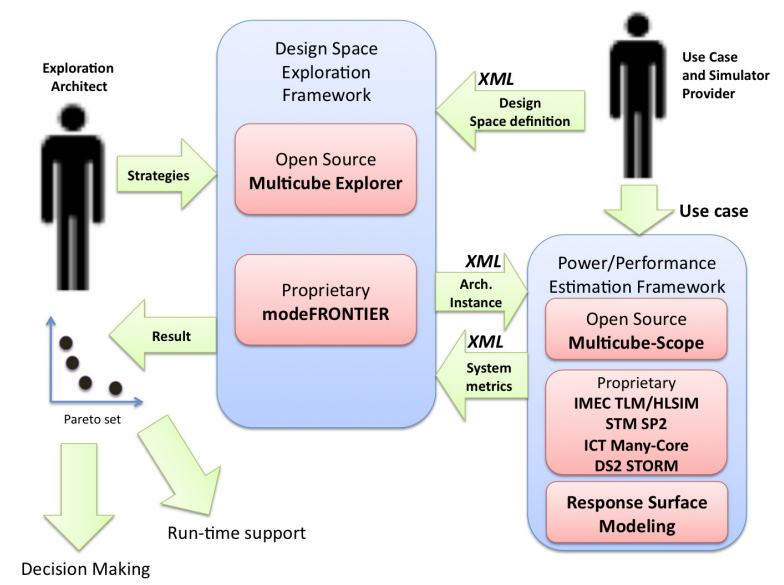

Fig. 1. The MULTICUBE design flow

parameters and performance and power estimation model for the target use case (model setup).

The Exploration Architect. (S)he is in charge of optimizing the configurable architecture by exploiting the automatic DSE framework. The exploration architect can directly interact with the DSE engine to set up exploration strategies, metrics and constraints.

The final exploration results (Pareto frontier) can be further pruned by a set of decision-making mechanisms. The solution set can be ordered according to some ranking criteria to derive the final candidate solution for the implementation. Besides, the Pareto frontier can then be used to support runtime management of the resources. This last feature has been already tested on an industrial use case and described in [1].

\section{Power And Performance Estimation FRAMEWORK}

The MULTICUBE project can easily manage a wide range of simulation technologies ranging from full-system cycleaccurate to application-level back-annotated functional simulation by using a standardized XML-based interface. The capability to span across several abstraction levels is fundamental to model cross-validation between different abstraction levels (e.g., high-level vs. TLM) or to provide mixed-level optimization strategies [2]. In the optimization literature, the simulator is also referred to as the solver.

The power and performance estimation tools can be grouped as open-source and proprietary:

Open Source Estimation Framework. The open source prototype tool is MULTICUBE-SCoPE and it is based on existing technology (SCoPE) [3] developed by University of Cantabria for performing HW/SW co-simulation. MULTICUBE SCoPE enables the definition of SystemC platform template models to evaluate performance and power consumption. MULTICUBE-SCoPE efficiency comes from the fact that performance and power estimations of the software side are performed at the application source code level by using back-annotation. The back-annotated software components are then linked to the hardware components by using a standard SystemC interfaces. This modeling style is called Timing Approximate. Software back-annotation avoids instruction set simulation therefore decreasing of several orders of magnitude the simulation time and maintaining a fairly good accuracy with respect to cycle-accurate simulations. MULTICUBESCoPE also provides some hooks for enabling $\mathrm{C} / \mathrm{C}++$ software code to invoke operating system primitives compliant with POSIX and MicroC/OS.

Proprietary Estimation Framework. These modeling and simulation tools span several abstraction levels. To simulate IMEC multimedia architecture based on the ADRES processor [4], two simulators are provided: a high-level simulator (HLSIM) and a SystemC-based platform simulator based on CoWare virtual prototyping environment. The target platform is shown in figure 2: it is composed of a variable number of processor nodes and memory nodes. All processor nodes contain an ADRES processor, IMEC proprietary VLIW processor and its scratch-pad local data (L1) memory. The processing nodes are connected to the memory nodes by a configurable communication infrastructure. It can be either a multi-layer AHB bus, which provides a full point-to-point connectivity, or a Network-on-Chip (NoC) model built with the CoWare AVF cross-connect IP.

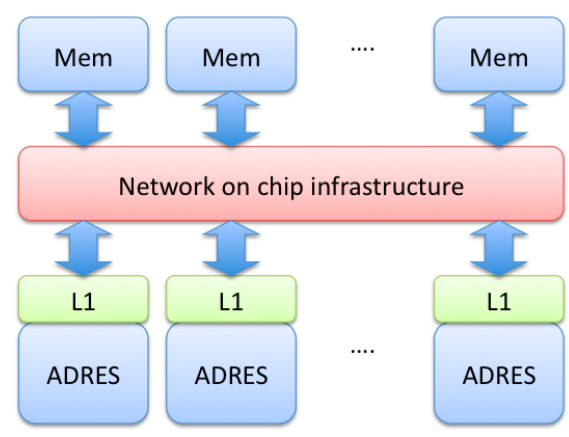

Fig. 2. IMEC ADRES platform.

From one side, IMEC HLSIM provides fast simulation at the source code level of parallel applications by providing support for timing annotation, thread spawning/joining, DMA transfers, FIFO communication and loop synchronization modeling. HLSim takes as an input the platform architecture description which contains information on the processors, the memory hierarchy structure, the timing figures and the power consumption of the other IP cores. For example, the timing information of the computation-intensive loops in the threads is measured on the compiled code simulator and is used as an input in HLSim. This means that the timing figures are instructionaccurate because they take all the compiler optimizations into account. On the other side, IMEC's CoWare simulator is based on cycle-accurate Transaction-Level Modeling (TLM) and provides a multi-processor platform template leveraging a Network-on-Chip (NoC) as interconnection model. The template links compute ADRES nodes (processing elements) and memory nodes together using a central communication back- 
bone. This model has been used for creating back-annotation data used in the HLSIM simulator and performing crossvalidation. Overall, we found an acceptable relative accuracy with a significant speed-up in simulation time (see Figure 3 , where the relative accuracy and speed of MULTICUBESCoPE technology has been added for comparison).

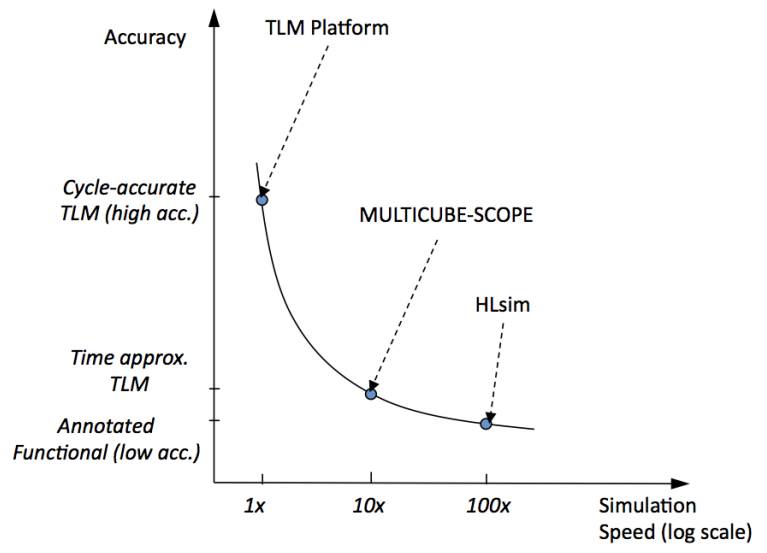

Fig. 3. Accuracy versus speed trade-off for several abstraction level simulators

An instruction set simulator has been used for SP2 superscalar processor provided from STMicroelectronics and for the many-core architecture from ICT. Both simulators expose program execution time and power consumption as system level metrics. More in detail, the ICT many-core architecture is a tiled MIMD machine composed of a bi-dimensional grid of homogeneous, general-purpose compute elements, called tiles. A 2D-mesh network architecture is used for connecting the cores to a non-shared memory sub-system.

Finally, as an example of control-oriented architecture, the DS2's STORM platform has been integrated in the framework. The platform is used to model a PLC (Programmable Logic Controller) technology with several implementation choices. For this platform, both Ethernet QoS and internal communication are considered as metrics.

\section{AdVAntages of AUtomatic DSE}

Multi-objective optimization heuristics and high-level simulation techniques can successfully contribute to dramatically decrease the exploration time, while guaranteeing a certain level of 'closeness' to the actual Pareto frontier. Reducing the exploration time reduces the time-to-market of the product and therefore the risk to miss market deadlines (with the associated economic loss).

Both conventional and automatic DSE starts from a definition of design-space (see Figure 4) representing the set of feasible architectural configurations. In a conventional DSE approach, the starting point is the definition of an initial model (model setup phase) of the target architecture. The enabling simulation technologies used for creating such a model can range from cycle-accurate SystemC up to annotated functional simulation, with variable ratio of accuracy with respect to speed. The exploration is done iteratively by subjective assumptions of the designer, who will edit manually the architectural simulator and modify a limited number of parameters per evaluation. The model simulation corresponds to a limited portion of the time of the overall exploration procedure. A larger amount of time is spent by the designer editing the configuration parameters and/or the model structure and analyzing the results. There is also an idle time (from the point of view of the use of computational resources) that lasts from the end of the simulation till the moment in which human operator is informed about it and handles the simulation tools to get the results.

In this case, the overall quality of the DSE is based on the designer's ability and past experience to assess the results and to move towards the next instance of the model to be simulated based on aggregate information resulting from the simulation campaign. The outcome (solution set) is thus highly dependent on the skills and past experience of the designer.

An automatic DSE flow uses numerical/objective criteria combined with efficient heuristics to drive the exploration. The basic assumption is that the model should be a configurable template model which can be automatically manipulated to generate any instance of the target design space. The model setup stage, thus, may be longer than in the conventional case. Given the configurable model template, the DSE tool will change systematically all the parameters at each step and will evaluate the best result based on robust optimisation heuristics. The automatic selection of the next configuration to be simulated (model selection) is typically faster than the conventional one because of it does not involve any overhead due to human intervention. The final output of the automatic DSE flow is a set of dominant configurations in the design space which are, with high probability, close to the actual Pareto set. Besides, all data concerning previous evaluations are stored in a structured database which can be automatically analyzed by using mathematical/statistical tools to derive aggregate information about the population of analyzed design points.

\section{Design Space EXPloration FrameWork}

The structure of the exploration framework is composed of an open-source optimization tool (MULTICUBE Explorer) and a proprietary multi-objective optimization tool (ESTECO's modeFRONTIER). The open-source tool has been designed from scratch to address embedded system exploration, while the proprietary tool is an existing tool for multi-disciplinary optimization which has been re-targeted to address discrete embedded system exploration. In general, DSE is an optimization process that takes into account a typical set of IP parameters that are associated with the memory system structure (e.g., cache size), the inherent parallelism of the processor (e.g., number of simultaneous tasks and the instruction issue width) and the on-chip interconnect configuration. The optimization problem involves either the maximization or minimization of multiple objectives (such as execution 


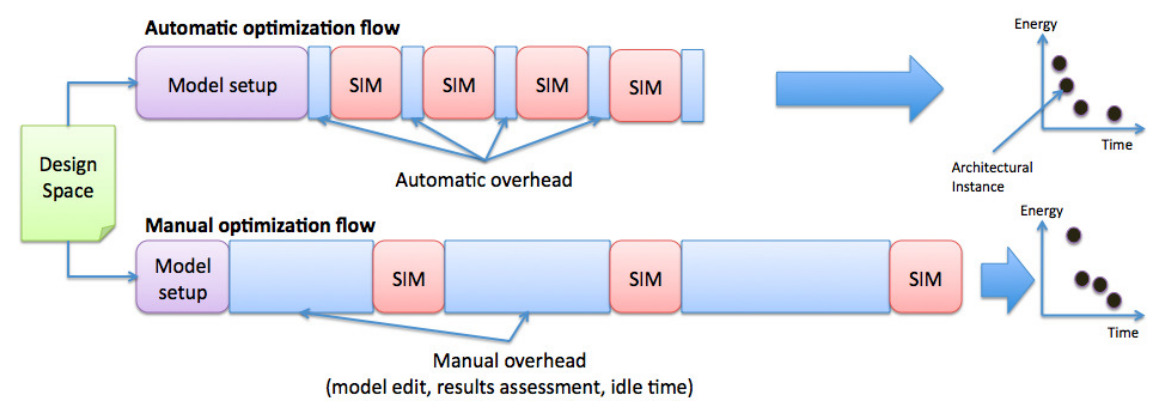

Fig. 4. Comparison between a manual (or conventional) design space exploration approach and an automatic approach.

time, power consumption, area, etc.) making the definition of optimality not unique [5].

In our context, the multi-objective optimization targets a set of $n$ system configuration parameters grouped on a configuration vector:

$$
\boldsymbol{a}=\left[\begin{array}{c}
a_{1} \\
\cdots \\
a_{n}
\end{array}\right] \in \boldsymbol{A}
$$

where $\boldsymbol{A}$ is usually a finite, discrete domain (subset of $N_{0}^{n}$ ). The multi-objective optimization problem is defined as a set of $m$ objective functions to be minimized (or maximized):

$$
\min _{\boldsymbol{a} \in \boldsymbol{A}} \phi(\boldsymbol{a})=\left[\begin{array}{c}
\phi_{1}(\boldsymbol{a}) \\
\cdots \\
\phi_{m}(\boldsymbol{a})
\end{array}\right] \in R^{m}
$$

subject to a set of $k$ constraints which, without loss of generality, can be expressed as:

$$
\chi(\boldsymbol{a})=\left[\begin{array}{c}
\chi_{1}(\boldsymbol{a}) \\
\cdots \\
\chi_{k}(\boldsymbol{a})
\end{array}\right] \leq\left[\begin{array}{c}
0 \\
\ldots \\
0
\end{array}\right]
$$

The set of feasible solutions of an optimization problem is defined as the feasible region:

$$
\Phi=\left\{\boldsymbol{a} \in \boldsymbol{A} \mid \boldsymbol{\chi}_{i}(\boldsymbol{a}) \leq 0,1 \leq i \leq k\right\}
$$

In single-objective optimization problems, the feasible set is totally ordered w.r.t. the function $\phi(\boldsymbol{a})$, thus a single exact solution $\boldsymbol{a}$ exists. When several objectives are defined, the feasible set is partially ordered through dominance relation and, thus, multiple exact solutions exist. The solution set is defined as the exact Pareto set of the problem. We say that $\phi$ dominates $\gamma$ when:

$$
\phi \prec \gamma=\left\{\begin{array}{c}
\phi_{i} \leq \gamma_{i}, \forall i=1, \ldots, m \text { and } \\
\phi_{i}<\gamma_{i} \text { for at least one } i
\end{array}\right.
$$

Given a subset of feasible configurations $\Omega \subseteq \boldsymbol{A}$, we define the Pareto set $\Psi$ associated to $\Omega$ as:

$$
\Psi(\Omega)=\left\{\boldsymbol{a} \mid\left(\begin{array}{c}
\boldsymbol{a} \in(\Phi \cap \Omega) \wedge \\
\neg \exists \boldsymbol{b} \in \Psi(\Omega) \text { s.t. } \boldsymbol{\phi}(\boldsymbol{b}) \prec \boldsymbol{\phi}(\boldsymbol{a})
\end{array}\right)\right\}
$$

The Pareto set $\Psi(\Phi)$ is the exact Pareto set of the problem. Based on set theory, the projection of $\Psi(\Phi)$ in the objective space is called the exact Pareto front.

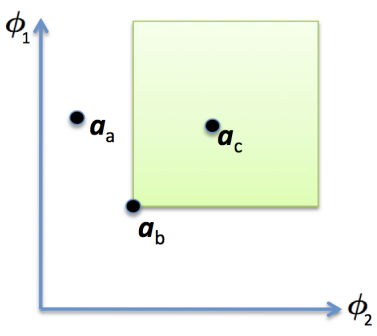

Fig. 5. Pareto Dominance, $\Omega=\left\{\boldsymbol{a}_{a}, \boldsymbol{a}_{b}, \boldsymbol{a}_{c}\right\}$. Point $\boldsymbol{a}_{c}$ is dominated by point $\boldsymbol{a}_{b}$.

Whenever the considered solution set $\Omega$ is a subset of the feasible solution space $\Phi$, the Pareto set $\Psi(\Omega)$ is called an approximate Pareto set of the problem.

Figure 5 shows a feasible set of solutions $\Omega=\Phi=$ $\left\{\boldsymbol{a}_{a}, \boldsymbol{a}_{b}, \boldsymbol{a}_{c}\right\}$ for an unconstrained minimization problem for $\left[\phi_{1}, \phi_{2}\right]$. Point $\boldsymbol{a}_{c}$ is dominated by point $\boldsymbol{a}_{b}$ since both $\phi_{1}\left(\boldsymbol{a}_{c}\right)$ and $\phi_{2}\left(\boldsymbol{a}_{c}\right)$ are greater than $\phi_{1}\left(\boldsymbol{a}_{b}\right)$ and $\phi_{2}\left(\boldsymbol{a}_{b}\right)$. In this case we thus have that $\Psi(\Omega)=\left\{\boldsymbol{a}_{a}, \boldsymbol{a}_{b}\right\}$.

The overall goal of multi-objective optimization heuristics is to identify approximate Pareto sets which are as near as possible to the exact Pareto set. For evaluating the quality of the approximate Pareto sets, a measure of the distance between the exact and the approximate Pareto sets can be introduced. In literature, many quality functions have been proposed to tackle this problem [6]. In the MULTICUBE project we leveraged the Average Distance from Reference Set (ADRS). The ADRS is used to measure the distance between the exact Pareto set $\Pi=\Psi(\Phi)$ and the approximate Pareto set $\Lambda=\Psi(\Omega)[7]$ :

$$
\operatorname{ADRS}(\Pi, \Lambda)=\frac{1}{|\Pi|} \sum_{\boldsymbol{a}_{R} \in \Pi}\left(\min _{\boldsymbol{a}_{A} \in \Lambda}\left\{\delta\left(\boldsymbol{a}_{R}, \boldsymbol{a}_{A}\right)\right\}\right)
$$

where $\delta$ is a measure of the normalized distance in the objective function space of two configurations:

$$
\delta\left(\boldsymbol{a}_{R}, \boldsymbol{a}_{A}\right)=\max _{j=1, \ldots, m}\left\{0, \frac{\phi_{j}\left(\boldsymbol{a}_{A}\right)-\phi_{j}\left(\boldsymbol{a}_{R}\right)}{\phi_{j}\left(\boldsymbol{a}_{R}\right)}\right\}
$$

The ADRS is usually measured in terms of percentage; the higher the ADRS, the worst is $\Lambda$ with respect to $\Pi$. 


\section{A. Multi-objective optimization heuristics}

In this section we present the multi-objective optimization heuristics implemented in MULTICUBE project and an analysis of the features carried out on an industrial architecture.

So far, the following optimisation algorithms have been implemented and analyzed:

- Standard algorithms: In this group we can find wellknown multi-objective optimisation algorithms that have been implemented by following the original specification. The group is composed of the NSGA-II [8] and MOGAII [9] algorithms.

- Enhanced algorithms: In this group we can find enhanced version of standard algorithms in order to deal with the specific discrete parameters addressed in MULTICUBE. The group is composed of the MOSA [10], ES and MOPSO [11] algorithms.

- New algorithms: In this group we can find all the algorithms that have been specifically defined in the MULTICUBE project for multi-objective optimization in the context of SoC design optimization. In this group we can find the MFGA (Evolutionary) [12] and APRS (Randomized) algorithms.

Evaluation of the algorithms. The algorithm validation shown here is based on the Low-Power Processor Use Case (Superscalar Processor SP2) delivered by STMicroelectronicsChina. The executable model for the design space exploration consists of sp2sim simulator, which is an instruction set simulator for the SP2 microprocessor design. The benchmark application selected is the 164.gzip application, based on the popular gzip compression algorithm. The design space is defined as a combination of 11 configuration parameters, among which out-of-order execution parameters (issue width, reorder buffer length, rename register number), cache sub-system (instruction and data cache configurations and load/store queue sizes), branch prediction (type and table size). For improving the validation speed, the number of input parameters was reduced to 7 by fixing the remaining ones to a constant value. The final design space of the problem is composed of 9,216 designs while the three objectives to be minimized are the total number of cycles, the power dissipation and the area.

A fair evaluation of non-deterministic algorithms requires several repeated runs without changing any parameter besides the random generator seed. Notwithstanding the relative small search space consisting of only 9,216 designs, very large variations can be observed in the algorithms behavior and a rigorous study needs to analyze also this aspect. Besides, preliminary tests were performed in order to identify nonsignificant parameters that have been then fixed to a constant value. Algorithms configuration parameters are usually problem-dependent. Typically, they depend on the user expectations: the optimal choices for the parameters that control the ratio between exploration and exploitation phase (e.g., the temperature schedule in the MOSA heuristic) are strictly related to the number of evaluations the user can afford. It was decided to tune these parameters considering the largest target (i.e. $50 \%$ of the design space, as described below) and accepting possible worse results in the partial samples. The evaluation process then proceeds checking at fixed numbers of explored points the quality of the non-dominated front found so far. The steps selected for the evaluation are: 184 designs (corresponding to about $2 \%$ of the design space), 461 (5\%), 922 (10\%), 1,843 (20\%), 2,765 (30\%), 3,686 (40\%) and $4,608(50 \%)$. Only the requests of evaluation of new designs were counted, since sometimes the algorithms requested the evaluation of an already evaluated design due to the inherent behavior of their random engines.

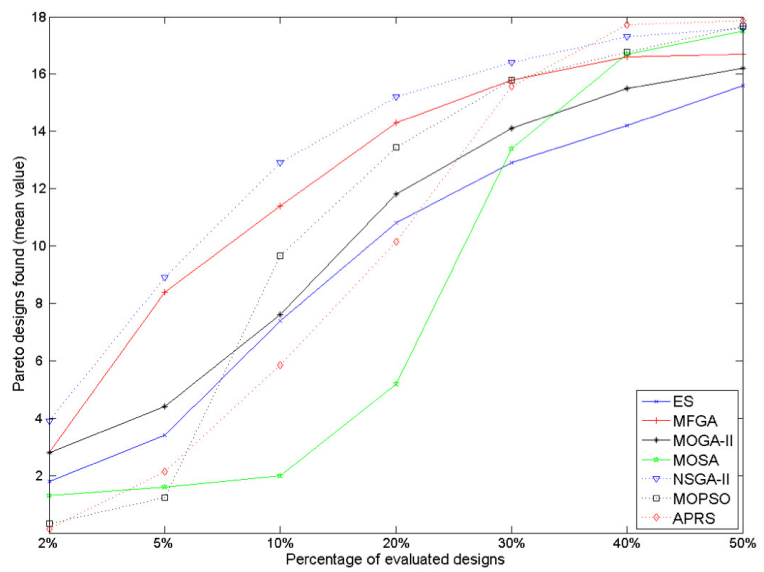

Fig. 6. Number of Pareto points found plotted against the percentages of evaluated designs.

Concerning all algorithms, we found that they easily reach an ADRS value below $2 \%$ evaluating $30 \%$ of the design space (lower ADRS corresponds to a better solution). This result can be considered very promising though variations in the slope of the lines for some algorithms are due to different 'behavioral' phases of the specific optimization process. The most clear example encountered in the project was the MOSA heuristic with its 'hot' and 'cold' phase. We point out also that a semiautomatic support is also provided by modeFRONTIER tool to choose the most suitable algorithm for the target design space.

Figure 6 shows the number of true Pareto points found by the algorithms at the specified percentage of design space exploration. MOSA is tuned to reach the top of the exploitation phase at $50 \%$ of evaluations and therefore its results are the worst up to $20-30 \%$, while at the end it is one of the most effective algorithms. APRS shows a similar behavior.

\section{B. Response surface modeling}

To further increase the efficiency of the exploration process, Response Surface Modeling (RSM) techniques have been introduced in the project. The basic idea is to reduce the number of simulations by defining an analytical response model of the system metrics based on a subset of simulations used as training set. Then the analytical expressions are used to predict the unknown system response. More in detail, RSM 
provide an approximation $\rho(\boldsymbol{a})$ of the given vector systemlevel metrics $\boldsymbol{\phi}(\boldsymbol{a})$. Each response $\rho(\boldsymbol{a})$ is trained with a set of observations derived from an actual simulation of a configuration $\boldsymbol{a}$. In the project, several RSM techniques have been implemented, among them Radial Basis Functions [13], Linear Regression [14], [15], Artificial Neural Networks [16] and Shepard's Interpolation. Every RSM presented dramatic speed-up in terms of evaluation. Besides, we found that a peculiar mathematical transformation of input training set known as Box-Cox $\lambda$ transform [14] has a great impact on the prediction accuracy. Figure 7 shows the average prediction accuracy of the linear regression response surface model on the system metrics of the ICT many-core architecture by varying the number of training samples, the model order and the $\lambda$ transform. We can note that, for a logarithmic or squared Box $\lambda$ transform ( $b o x=\log$ and $b o x=0.5$ ) and a second order linear regression model, we have less than $10 \%$ relative error with as few as $9 \%$ of the design space used as a training set.

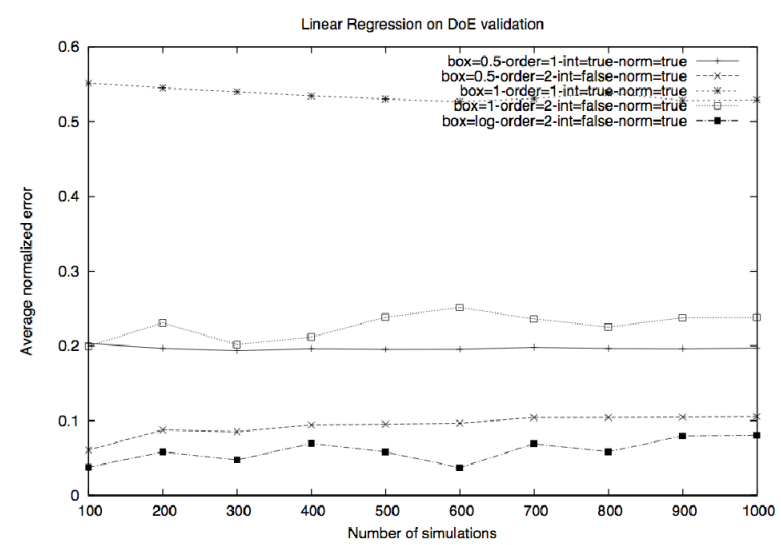

Fig. 7. Linear regression prediction accuracy versus the number of training samples (100 samples $\simeq 9 \%$ of the design space).

\section{CONCLUSIONS}

In this paper we presented the fundamental problems addressed and the main achievements of the European Project MULTICUBE. In particular, we presented the definition of an automatic design space exploration framework combined with a power/performance estimation framework to support the design of next generation many-core architectures.

\section{ACKNOWLEDGEMENTS}

We would like to gratefully acknowledge our EC Project Officer, Panagiotis Tsarchopoulos and our reviewers: Alain Perbost, Andrzej Pulka and Kamiar Sehat for their valuable comments and guidance.

Prabhat Avasare, Geert Vanmeerbeeck, Chantal Ykman and Maryse Wouters are also associated with Interdisciplinary Institute for BroadBand Technology, Belgium (IBBT), B-9050 Gent, Belgium.

\section{REFERENCES}

[1] G. Mariani, P. Avasare, G. Vanmeerbeeck, C. Ykman-Couvreur, G. Palermo, C. Silvano, and V. Zaccaria. An industrial design space exploration framework for supporting run-time resource managemen on multi-core systems. In Proceedings of DATE 2010: IEEE Design, Automation and Test Conference in Europe, pages 196-201, Dresden, Germany, March 2010.

[2] G. Mariani, G. Palermo, C. Silvano, and V. Zaccaria. Multiprocessor system-on-chip design space exploration based on multi-level modeling techniques. In Proceedings of IEEE IC-SAMOS'09 - International Conference on Embedded Computer Systems: Architectures, MOdeling, and Simulation, pages 118-124, Samos, Greece, July 2009.

[3] Hector Posadas, Juan Castillo, David Quijano, Victor Fernandez, Eugenio Villar, and Marcos Martinez. SystemC platform modeling for behavioral simulation and performance estimation of embedded systems. Behavioral Modeling for Embedded Systems and Technologies: Applications for Design and Implementation, pages 219-243, 2010.

[4] B. Mei, B. Sutter, T. Aa, M. Wouters, A. Kanstein, and S. Dupont. Implementation of a coarse-grained reconfigurable media processor for avc decoder. J. Signal Process. Syst., 51(3):225-243, 2008.

[5] Hwang, C. L. and Masud, A. S. M. . Multiple Objective Decision Making - Methods and Applications: A State-of-the-Art Survey, volume 164. Lecture Notes in Economics and Mathematical Systems, SpringerVerlag, Berlin-Heidelberg, 1979.

[6] T. Okabe, Y. Jin, and B.off. A critical survey of performance indices for multi-objective optimization. In Proceedings of the IEEE Congress on Evolutionary Computation, pages 878-885, 2003.

[7] Jaszkiewicz A. Czyak P. Pareto simulated annealing - a metaheuristic technique for multiple-objective combinatorial optimisation. Journal of Multi-Criteria Decision Analysis, (7):34-47, April 1998.

[8] K. Deb, S. Agrawal, A. Pratab, and T. Meyarivan. A Fast and Elitist Multi-Objective Genetic Algorithm: NSGA-II. Proceedings of the Parallel Problem Solving from Nature VI Conference, pages 849-858, 2000.

[9] Carlo Poloni and Valentino Pediroda. GA coupled with computationally expensive simulations: tools to improve efficiency. In D. Quagliarella, J. Périaux, C. Poloni, and G. Winter, editors, Genetic Algorithms and Evolution Strategies in Engineering and Computer Science. Recent Advances and Industrial Applications, chapter 13, pages 267-288. John Wiley \& Sons, Chichester, UK, 1998.

|[10] K.I Smith, R.M Everson, J.E Fieldsend, C Murphy, and R Misra. Dominance-based multiobjective simulated annealing. Evolutionary Computation, IEEE Transactions on, 12(3):323-342, 2008.

|[11] G. Palermo, C. Silvano, and V. Zaccaria. Discrete particle swarm optimization for multi-objective design space exploration. In Proceedings of DSD 2008: IEEE Euromicro Conference on Digital System Design Architectures, Methods and Tools, pages 641-644, Parma, Italy, September 2008.

[12] A. Turco and C. Kavka. MFGA: A genetic algorithm for complex realworld optimization problems. In Proceedings of BIOMA 2010, the 4th International Conference on Bioinspired Optimization Methods and their Applications, Lubiana, Slovenia, To appear in May 2010.

I[13] P. J. Joseph, Kapil Vaswani, and Matthew J. Thazhuthaveetil. A predictive performance model for superscalar processors. In MICRO 39: Proceedings of the 39th Annual IEEE/ACM International Symposium on Microarchitecture, pages 161-170, Washington, DC, USA, 2006. IEEE Computer Society.

I[14] P.J Joseph, Kapil Vaswani, and M.J Thazhuthaveetil. Construction and use of linear regression models for processor performance analysis. High-Performance Computer Architecture, 2006. The Twelfth International Symposium on, pages 99- 108, 2006.

[15] B. C. Lee and D. M. Brooks. Accurate and efficient regression modeling for microarchitectural performance and power prediction. Proceedings of the 12th international conference on Architectural support for programming languages and operating systems, 40(5):185-194, 2006.

I[16] C. Bishop. Neural Networks for Pattern Recognition. Oxford University Press, 2002. 